\title{
Neutrophil Viability as a Clinical Outcome Marker in Mechanically Ventilated Critically Ill Trauma Patients: A Case Series
}

\author{
Orsolya Benedek ${ }^{1 *}$, Mihaly Veres ${ }^{2}$, Minodora Dobreanu ${ }^{1}$ \\ 1 University of Medicine and Pharmacy of Tirgu Mures, Romania \\ 2 County Clinical Emergency Hospital Tirgu Mures, Romania
}

\begin{abstract}
Background: Trauma in its early stages leads to an acute inflammatory condition affecting all cellular lines. Neutrophil granulocytes make up the largest population of human white blood cells and are fundamental to the innate immune system. The objective of our pilot study was to evaluate neutrophil death and viability alterations in critically ill trauma patients in correlation with their clinical outcome.
\end{abstract}

Material and method: Critical ill trauma patients were enrolled in the study. In order to assess alterations in cellular death, blood samples were drawn using EDTA containing tubes and analyzed in the first twenty four hours after admission, then after forty eight and seventy two hours. Annexin $\mathrm{V}$ was used as a marker for apoptotic cells and propidium iodide for necrotic cells.

Results: The first two cases exhibited an increase in cellular viability by the second day as shown by a small increase in neutrophil apoptosis and a decrease in neutrophil necrosis. These patients progressed to a positive clinical outcome. The second two cases showed slight modifications in either physiological or pathological cellular death, and increasing levels of cellular necrosis. These patients progressed to a negative clinical outcome.

Conclusions: These cases suggest that neutrophil cell viability and death were associated with the patient's clinical outcome.

Keywords: critical, trauma, neutrophil, viability, marker

Received: 10 June 2015 / Accepted: 05 July 2015

\section{INTRODUCTION}

Trauma in its early stages is an acute condition, affecting all cellular lines, activating the innate immune system by releasing inflammatory mediators and other biochemically active agents. Neutrophil granulocytes make up the largest population of human peripheral white blood cells and are fundamental to the innate immune system. Chemotaxis permits neutrophil migration from the circulating blood to the site of affected tissue. Their circulating half-life in vivo of neutrophil is approximately six to eight hours, after which they ordinarily suffer apoptotic cell death conditions. The type of acute pathology is highly influential and depending on many associated factors, neutrophilhalf-life may either be prolonged or result in to untimely necrosis [1].

Apoptosis is programmed cell death, considered physiological and accompanied by several cellular changes. On initiation of apoptosis, phosphatidylserine, which is located on the inner surface of the plasma membrane, is translocated to the outer surface of the cell. Annexin V is a protein with high affinity for phosphatidylserine $[2,3]$.

Necrosis is premature cell death, being considered a pathological process. Propidium iodide is a fluorescent molecule which binds to nucleic acids of necrotic cells, 
allowing detection by flow cytometry. These markers allow the differentiation of viable from necrotic cells $[3,4]$.

\section{Objective}

The objective of the pilot study was to evaluate changes in neutrophil death and viability in critically ill trauma patients, and to correlate this with their clinical outcome.

\section{MATERIAL AND METHOD}

We enrolled critical trauma patients in a pilot study, with the approval of the Tirgu Mureş Emergency Clinical County Hospital's Ethics Committee, in order to determine peripheral blood cellular death modifications as a possible prognostic marker. All the patients were admitted in the Tirgu Mureş Emergency Clinical County Hospital's Intensive Care Clinic. We chose this specific group, due to their mechanically ventilated status during our biological monitoring.

In all the cases, patient relatives agreed on the use of their biological samples for the purpose of this study. Blood samples were analysed in the Tirgu Mureş Emergency Clinical County Hospital's Central Laboratory. A total blood count was performed before each flow cytometric analysis, to ensure the necessary number of cells needed for gating. The blood was prepared for flow cytometric analysis within five minutes of sampling in order reduce ex-vivo cell death. We set up a sampling protocol, consisting of flowcytometric whole blood analysis. Annexin V-FITC Apoptosis Detection Kit II (BD Biosciences) was used to detect apoptosis and Propidium Iodide staining solution (BD PharmingenTM) to detect necrosis. Blood samples were drawn (using K-EDTA containing tubes) and analysed in the first twenty-four hours after admission to the intensive care unit and thereafter at forty eight hours and seventy two hours.

CaliBRITE beads and FACSComp software (BD Biosciences, San Jose, California, USA) were used for setting the photomultiplier tube voltages, fluorescence compensation and for checking instrument sensitivity prior to use. Acquisition and analysis was achieved by CellQuest software using a BD FACS CaliburTM flow cytometer (BD Biosciences, San Jose, California, USA). The cellular light-scatter signals and two fluorescence signals were analysed in list mode at a channel resolution of 1024, with forward scatter as trigger parameter. All data were displayed as two-color dot plots and histogram statistics.

\section{RESULTS}

\section{Case 1}

A fifty two year old female patient was admitted to the Intensive Care Clinic after a car accident with severe cerebral and facial trauma, parietal scalp lesions, chest trauma and lung contusions, X-XII rib fractures, bilateral haemothorax, abdominal trauma, haemoperitoneum with mesenteric rupture, pelvic fracture with retro-peritoneal haematomas. No known pre-existing conditions were elicited. Shortly after admission an exploratory laparotomy was performed in order to allow suturing of the mesenteric rupture and lavage of the haemo-peritoneum. Upon admission to the Intensive Care Clinic, the patient's status was critical. Without reversal of anaesthesia, she was intubated and mechanically ventilated by bi-level positive airway pressure ventilation, to render her haemodynamically stable. Blood gas values and electrolytes were within the normal range. The white blood cell count showed 10300 cells/ $\mu$ l with an $88 \%$ neutrophil count.

The first blood sample for flow cytometric analysis was drawn eight hours after admission and showed the level of viable neutrophils in the circulating blood to be $0.07 \%$. There were no apoptotic neutrophil forms, $0.9 \%$ intermediate cells and 99\% necrotic neutrophils.

On the second day, the blood was sampled exactly twenty four hours after the first sample. The number of viable neutrophils in the circulating blood reached $0.1 \%$, with no apoptotic cell forms present. The intermediate cell types increased to $2.7 \%$ and necrotic cells decreased to $97.2 \%$. The patient remained intubated and mechanically ventilated in continuous positive airway pressure mode, and had a spontaneous breathing rate of twenty breaths/minute. All signs were suggestive of an improvement in the patient's clinical status. She had a Glasgow Coma Score of 15 points and a FOUR score of 13 points. White blood cell numbers s decreased to 9500 cells/ $\mu$ lwith an $84.8 \%$ neutrophil count.

On the third day again exactly twenty four hours after the previous sample,blood was obtained for flow cytometric analysis. At this time period, there was an increase in viable neutrophils to $21 \%$, accompanied by $0.3 \%$ of apoptotic cell forms. The intermediate cell population had decreased $\mathrm{f} 2.8 \%$ and necrotic neutrophils to $75.3 \%$. The patient's clinical status showed further improvement, but mechanical ventilation was 
still deemed necessary. The white blood cell count was down to 7980 cells/ $\mu$ l with $84.8 \%$ being neutrophils.

The patient was taken off mechanical ventilation on the fifth day after admission having shown improved clinical and biological status, normal white blood cell and neutrophil count, and a positive clinical outcome.

\section{Case 2}

A fifty two year old male patient was admitted to the Intensive Care Clinic, after a car accident, presenting with severe cerebral and facial trauma, cerebral contusions, subdural haematoma, subarachnoid bleeding, zygomatic arch fracture and no known pre-existing conditions. Upon admission to the Intensive Care Clinic, the patient's status was critical. While still under residual sedation administered in the emergency department, he was intubated and mechanically ventilated in bi-level positive airway pressure mode, to render her haemodynamically stable. Blood gas values showed a slight hypercapnia of $48 \mathrm{mmHg}$, with electrolytes in the normal range. The white blood cell count showed 10400 cells $/ \mu$ of blood with a $79 \%$ neutrophil count.

The first blood sample for flow cytometric analysis was drawn 7 hours after admission, and showed viable neutrophils in the circulating blood to be $0.3 \%$,with no apoptotic neutrophil forms, $1.6 \%$ intermediate cells and $98.1 \%$ necrotic neutrophils.

By the second day, the number of viable neutrophils reached $0.4 \%$ in the circulating blood. No apoptotic cell forms present but the intermediate forms increased to $4.8 \%$ and necrotic cells decreased to $94.8 \%$. The patient's clinical status was improving, but he was maintained under sedation due to spontaneous breathing pattern difficulties. He was intubated and mechanically ventilated in bi-level positive airway pressure ventilation. His blood gas analyses normalized. The white blood cells decreased to 9800 cells/ $\mu$ l of blood with a $77 \%$ neutrophil count.

On the third day, the viable neutrophil population showed an increase to $1.7 \%$, and the first apoptotic cell forms were detected, being $0.4 \%$ of the total population. The intermediate cell population was $7.1 \%$, and there was a decrease in necrotic neutrophils to $90.9 \%$. The patient's clinical status showed further improvement but the first attempt to take him off mechanically ventilated failed on the third day. The white blood cell count was down to $9280 \mathrm{cells} / \mu \mathrm{l}$ with a neutrophil count of $75 \%$.
The patient was taken off mechanical ventilation on the fifth day following an improvement in his clinical and biological status, normal white blood cell and neutrophil count.

\section{Case 3}

An eighty year old male patient was admitted to the Intensive Care Clinic after a car accident having had resuscitated cardiac arrest (pulseless electrical activity) following the traumatic event. He presented with severe cerebral and facial trauma, fracture of the right humerus and pre-existing left ventricular dysfunction. Upon admission to the Intensive Care Clinic, the patient's status was critical. While under sedation administered in the emergency department, he was intubated and mechanically ventilated in bi-level positive airway pressure mode, with vasoactive support due to existing haemodynamic instability. Blood gas values showed severe respiratory acidosis with a $\mathrm{pH}$ of 7.0, a hypoxia of $49 \mathrm{mmHg}$ and a hypercapnia of $50 \mathrm{mmHg}$ including low sodium and high potassium levels. Lactate levels were highly increased at $5.9 \mathrm{mmol} / \mathrm{l}$. The white blood cell count showed $20100 \mathrm{cells} / \mu \mathrm{l}$. The pre-existing heart conditions were medically treated according to the recommendations of the attending cardiologist.

The first blood sample for cell staining and flow cytometric analysis was drawn ten hours after admission. There were $0.9 \%$ of viable neutrophils in the circulating blood, no apoptotic neutrophil forms, 1.4\% intermediate cells and $97.6 \%$ necrotic neutrophils.

On the second day, viable neutrophils dropped to $0.4 \%$ in the circulating blood, with no apoptotic cell forms present. However intermediate forms increased to $2.1 \%$ with an insignificant decrease in necrotic cells to $97.5 \%$. The patient's clinical status was not improving. He remained intubated and mechanically ventilated and hemodynamical stability was achieved only with positive inotrope and vasoactive drugs. His blood gas analyses showed a decreased hypoxia and hypercapnia, but potassium and lactate levels were still high. The white blood cells decreased to 9000 cells/ $\mu$ l of blood.

On the third day the viable neutrophil population showed a very small increase to $0.5 \%$, though this remained lower than the first measured value. No apoptotic cell forms were detected. The intermediate cell population was at $4.7 \%$ with a slight decrease in necrotic neutrophils to $95.8 \%$. The patient's clinical status showed no further improvement and it was not possible to take him off mechanical ventilation. The white 
blood cell count showed an increase to 10560 cells/ $\mu$ of blood with a neutrophils at $67.9 \%$.

The patient had several unsuccessful attempts to take him off mechanical ventilation. No improvement in clinical and biological status was achieved in the long term. The clinical outcome was negative.

\section{Case 4}

A seventy six year old male patient was admitted to the Intensive Care Clinic after a trauma from falling. He presented with severe cerebral and facial trauma, zygomatic arch fracture, chest contusion and multiple bilateral rib cage fractures, bilateral haemo-pneumothorax, pulmonary contusions and sternum and scapula fractures. Upon admission to the Intensive Care Clinic, the patient's status was critical. He was still under sedation administered in the emergency department. He was intubated and mechanically ventilated in bi-level positive airway pressure ventilation and was haemodynamically stable. Blood gas values and electrolytes were within the normal range. The white blood cell count showed 10230 cells/ $\mu$ l with $83.5 \%$ neutrophils.

The first blood sample for cell staining and flow cytometric analysis was drawn twenty hours after admission and it showed viable neutrophils in the circulating blood to be $0.05 \%$. There were no apoptotic neutrophil forms, 3.8\% intermediate cells and $96.1 \%$ necrotic neutrophils.

By the second day, the number of viable neutrophils showed a slight increase to $0.1 \%$ in the circulating blood, with no apoptotic cell forms present, and the intermediate forms showed a decrease to $3.4 \%$, with a slight increase in necrotic cells noted at $96.5 \%$. The patient's clinical status was not improving. He was con-

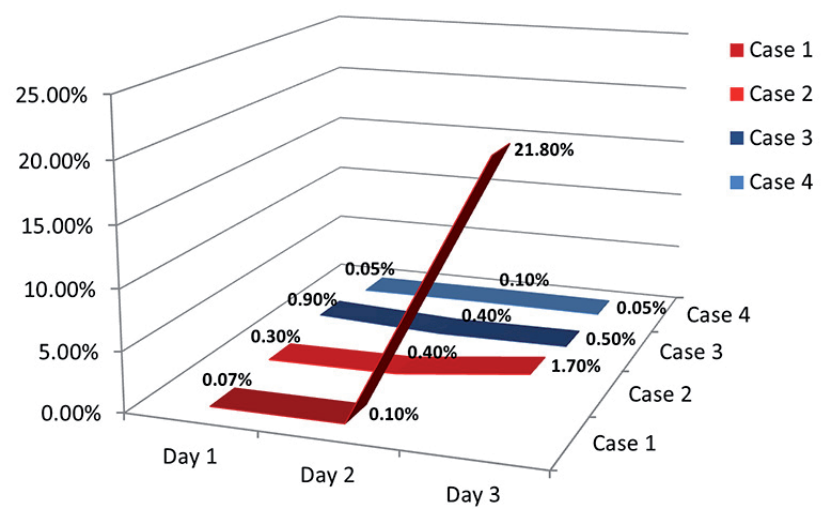

Figure 1. Neutrophil viability changes in all cases tinuously sedated, and no attempts were made to take him off mechanical resuscitation. His blood gas analyses showed no changes. The white blood cells decreased to 7800 cells $/ \mu \mathrm{l}, 84.2 \%$ being neutrophils.

On the third day, the viable neutrophil population dropped back to $0.05 \%$, the first measured value. No apoptotic cell forms were detected. The intermediate cell population was $12.2 \%$, with a decrease in necrotic neutrophils to $87.7 \%$. The patient's clinical status showed no further improvement. It was not possible to take him off mechanical ventilation. White blood cell count showed an increase to 11820 cells/ $\mu$ l, $85.7 \%$ being neutrophils.

During the course of treatment, no attempts were made to remove mechanical ventilation. No improvement in clinical and biological status was achieved in the long term. The clinical outcome was negative.

By the third day of biological monitoring, in all positive clinical outcome cases, the neutrophil cell population showed a significant increase in viability of over $1 \%$ (Figure 1).

\section{DISCUSSION}

Neutrophil granulocytes are the first line of response in the non-specific immune acute conditions. In critically ill patients they can quickly reach a peak level and given their short life span, the reservoir may be quickly depleted by pathological cell death due to necrosis.

In the presented cases, we found that determinations of neutrophil apoptosis and necrosis on three consecutive days, correlated with the patient's eventual clinical outcome.

Although the dominant form of cell death in acute pathological states is necrosis, likewise shown in our cases, cell apoptosis should not be overlooked. Morrison [5] conducted a study on forty one trauma patients with haemorrhagic shock, using the nucleosome ELISA technique to determine the level of apoptosis of peripheral blood neutrophils in order to correlate it with the reduced incidence of infection. The results demonstrated that peripheral leukocyte apoptosis occurs in set populations, and that it is higher in those patients who tend not to develop infectious complications. Furthermore, the results showed that those patients who did not develop infection had a two and a half times higher leukocyte apoptosis level compared to those who did develop infection [5]. 
In our study group the apoptotic cell levels were small, but they were completely absent in the cases that showed no improvement in clinical outcome. None of the four cases in the present series had documented infections, but all the patients received prophylactic antibiotic treatment.

In a review on neutrophil cell death in response to infection, it was concluded that although apoptosis has been the major focus of investigation, necrosis also plays a significant role in this critical situation [6].

Although none of our cases had documented clinical signs of infection per se, the biological signs of the inflammatory processes were present, shown by high levels of white blood cells with higher than normal neutrophil levels.

Savill [7] and Li [8] both stipulated that necrotic cells trigger the immune system and that the presence of necrotic cells, especially neutrophils, is pro-inflammatory. These findings were later challenged by Miles et al [9] who described $\alpha$-defensins as active anti-inflammatory factors released by apoptotic/necrotic neutrophils, as shown in their studies. Their data showed that necrotic human neutrophils are in fact, anti-inflammatory, thus, neutrophil necrosis at sites of inflammation initiates its resolution rather than trigger it.

In all samples obtained from the four present cases, necrosis was the prominent type of neutrophil cell death. However, reduction in the levels of necrotic cell forms and an increase in the level of viable cell forms was associated with a better clinical outcome, this association being stronger than an increase or even the maintenance in necrotic cell forms levels.

The modification in intermediate cell forms may be caused by the fact that sometimes necrotic cells can exteriorize phosphatydilserine, and can be mistaken for apoptotic cells. To avoid or reduce a false positive reaction, propidium iodide was used as a staining solution as it specifically stains necrotic cells [4].

A limitation of the present study is related to the blood sampling. Whole blood has to be analyzed in a matter of minutes after sampling to exclude ex vivo cell necrosis

This was accomplished in these four cases, but is accepted as a difficult during normal clinical circumstances. Another major limitation is expense related, given the high costs of the apoptosis detection kits and adjacent laboratory equipment.

\section{- CONCLUSIONS}

In this case series, neutrophil cell viability and death, be it apoptosis or necrosis, correlated with the patient's clinical outcome. Patients with a favourable clinical outcome showed increase in cellular viability by the second day in the Intensive Care Clinic, with a notable increases in viability, a small increases in neutrophil apoptosis and a decreases in neutrophil necrosis. Patients who did not have a positive clinical outcome showed slight modifications in physiological or pathological cell death, and static or increasing levels of cellular necrosis. The results indicated that a larger scale study was warranted and this is now in progress.

\section{ACKNOWLEDGEMENT}

This paper is supported by the Sectorial Operational Programme Human Resources Development (SOP HRD), financed from the European Social Fund and by the Romanian Government under the contract number POSDRU/159/1.5/S/133377/.

\section{DREFRENCES}

1. Hietbrink F, Koenderman L, Rijkers GT, Leenen LPH. Trauma: the role of the innate immune system. World J Emerg Surg, 2006;1:15.

2. Zhang G, Gurtu V, Kain SR, Yan G. Early detection of apoptosis using afluorescent conjugate of Annexin V. Bio Techniques. 1997;23:525-31.

3. Ulukaya E, Acilan C, Ari F, Ikitimur E, Yilmaz Y. A glance at the methods for detection of apoptosis qualitatively and quantitatively. Turk J Biochem. 2011;36:261-9.

4. Rieger AM, Nelson KL, Konowalchuk JD, Barreda DR. Modified Annexin V/Propidium lodide apoptosis assay for accurate assessment of cell death. J Vis Exp. 2011;50: 2597.

5. Morrison AC, Moran A, Patel S, Del Pilar.Huby.Vidaurre M, Carrick MM, Tweardy DJ. Increased apoptosis of peripheral blood neutrophils with reduced incidence of infection in trauma patients with hemorrhagic shock. J Infect. 2013;66:87-94.

6. Iba T, Hashiguchi N, Nagaoka I, Tabe Y, Murai M. Neutrophil cell death in response to infection andits relation to coagulation. J Intensive Care. 2013,1:13.

7. Savill J, Dransfield I, Gregory C, Haslett C. A blast from the past:clearance of apoptotic cells regulates immune responses. Nat Rev Immunol. 2002;2:965-75.

8. Li M, Carpio DF, Zheng Y, et al. An essential role of the NF-B/ Toll-like receptor pathwayin induction of inflammatory and tissue-repair gene expression by necrotic cells. J Immunol. 2001;166:7128-35.

9. Miles K, Clarke DJ, Lu W, et al. Dying and necrotic neutrophils are anti-inflammatory secondary to the release of $\alpha$-defensins. J Immunol. 2009;183:2122-32. 